\title{
Monitoria de enfermagem da disciplina de semiologia e semiotécnica: um relato de experiência
}

\author{
Nursing monitoring of the discipline of semiology and semiotechnology: an experience report \\ Seguimiento de enfermería de la disciplina de semiología y semiotecnia: informe de una experiencia
}

Recibido: 02/03/2021 | Revisado: 09/03/2021 | Acepto: 12/03/2021 | Publicado: 19/03/2021

Mariana Silva Souza

ORCID: https://orcid.org/0000-0003-1920-5367 Cristo Faculdade do Piauí, Brasil

E-mail: marianasouza_s@hotmail.com

Marcos Roberto Nascimento Sousa

ORCID: https://orcid.org/0000-0003-1634-5276 Cristo Faculdade do Piauí, Brasil

E-mail: marcosrobertoenfpi@gmail.com

Luciana Aparecida da Silva

ORCID: https://orcid.org/0000-0002-6653-3817 Cristo Faculdade do Piauí, Brasil

E-mail: nurselucianasilva@hotmail.com

Daniel Lopes Araújo

ORCID: https://orcid.org/0000-0002-1625-0368

Universidade Federal de Pernambuco, Brasil

E-mail: araujodanielopes@gmail.com

Sabrina Beatriz Mendes Nery

ORCID: https://orcid.org/0000-0002-8254-0152

Cristo Faculdade do Piauí, Brasil

E-mail: sabrinaanery2019@gmail.com

José Eufrazino Júnior

ORCID: https://orcid.org/0000-0002-4093-7952 Cristo Faculdade do Piauí, Brasil

E-mail: eufrazinojunior@gmail.com

Iani Clara Oliveira do Nascimento

ORCID: https://orcid.org/0000-0002-2131-352X

Cristo Faculdade do Piauí, Brasil

E-mail: ianioliveira46@gmail.com

Emilly da Silva Pereira

ORCID: https://orcid.org/0000-0003-4263-2316 Cristo Faculdade do Piauí, Brasil

E-mail: emillypereira63@gmail.com

Luís Henrique Araújo Andrade

ORCID: https://orcid.org/0000-0002-3504-0710 Cristo Faculdade do Piauí, Brasil

E-mail: luishenriqueenf22@gmail.com

Emanuelly Emyly da Costa

ORCID: https://orcid.org/0000-0001-9958-6707

Cristo Faculdade do Piauí, Brasil

E-mail: e.emyly@hotmail.com

Marcelo da Silva

ORCID: https://orcid.org/0000-0003-0421-1924

Cristo Faculdade do Piauí, Brasil

E-mail: marceloenf21@gmail.com

Kayco Damasceno Pereira

ORCID: https://orcid.org/0000-0002-3112-1193

Cristo Faculdade do Piauí, Brasil E-mail: kaycopereir@gmail.com

Luís Carlos Lopes Barbosa

ORCID: https://orcid.org/0000-0002-4272-1290

Cristo Faculdade do Piaú, Brasil

E-mail: carlosloppess@ outlook.com

\section{Resumo}

A monitoria é uma atividade que oportuniza a participação do aluno-monitor em momentos teóricos e práticos juntamente aos discentes com o objetivo de aprofundar o conteúdo e em busca de um desenvolvimento associado à prática docente. A disciplina de Semiologia e Semiotécnica por sua parte, abrange os procedimentos técnicos 
executados no laboratório e no carregar de toda a vida do profissional enfermeiro. Entretanto, o monitor faz-se um facilitador da aquisição de conhecimento do outro acadêmico. O presente estudo tem como objetivo relatar a experiência vivenciada frente a monitoria de Semiologia e Semiotécnica por graduandos do curso de enfermagem da Cristo Faculdade do Piauí. Trata-se de um estudo descritivo, do tipo relato de experiência. A monitoria ocorreu no período de agosto a dezembro de 2020, correspondendo ao semestre 2020/2. Foram momentos por intermédio da plataforma remota Google Meet diante dos discentes participantes. Os momentos de monitoria proporcionaram a criação de vínculo entre os monitores e alunos beneficiados pelo programa de monitoria, e isso facilitou o processo de ensino-aprendizagem da disciplina em questão. No decorrer da monitoria foi possível por intermédio da revisão contínua dos conteúdos a criação de uma base sólida de conhecimento, bem como pela busca constante de atualização de informação e o uso de tecnologias, assim sendo inferido a tamanha importância do programa de monitoria para os discentes da faculdade. A experiência representou-se como uma atividade relevante para os monitores, uma vez que possibilitou o crescimento acadêmico, profissional e pessoal, assim como podemos observar diversos ganhos ao público participante.

Palavras-chave: Estudantes de enfermagem; Ensino; Educação em enfermagem; Educação a distância.

\begin{abstract}
The monitoring is an activity that provides an opportunity for the student-monitor to participate in theoretical and practical moments with the students in order to deepen the content and in search of a development associated to the teaching practice. The discipline of Semiology and Semiotechnology, on its part, covers the technical procedures performed in the laboratory and throughout the life of the professional nurse. However, the monitor becomes a facilitator of the other student's acquisition of knowledge. The present study aims at reporting on the experience of the Semiology and Semiotechnology monitoring by undergraduate students of the nursing course of the Cristo Faculdade do Piauí. This is a descriptive study, of the experience report type. The monitoring occurred from August to December 2020, corresponding to the semester 2020/2. These were moments through the remote platform Google Meet in front of the participating students. The moments of monitoring provided the creation of bonds between the monitors and students benefited by the monitoring program, and this facilitated the teaching-learning process of the subject in question. Through the continuous review of the contents, it was possible to create a solid base of knowledge, as well as the constant search for updated information and the use of technology, thus inferring the great importance of the monitoring program for the students of the university. The experience represented a relevant activity for the monitors, since it allowed for academic, professional, and personal growth, as well as several gains for the participating public.
\end{abstract}

Keywords: Nursing students; Teaching; Nursing education; Distance education.

\title{
Resumen
}

El seguimiento es una actividad que brinda la oportunidad al alumno-monitor de participar en momentos teóricos y prácticos con los alumnos para profundizar en los contenidos y en busca de un desarrollo asociado a la práctica docente. La disciplina de la Semiología y la Semiótica por su parte, abarca los procedimientos técnicos realizados en el laboratorio y en la realización a lo largo de la vida del profesional de la enfermería. Sin embargo, el monitor se convierte en un facilitador de la adquisición de conocimientos del otro alumno. El presente estudio tiene como objetivo relatar la experiencia vivida frente al Monitor de Semiología y Semiotecnia por estudiantes de pregrado del curso de enfermería de la Cristo Faculdade do Piauí. Se trata de un estudio descriptivo, de tipo informe de experiencia. El seguimiento se realizó en el periodo comprendido entre agosto y diciembre de 2020, correspondiente al semestre 2020/2. Fueron momentos a través de la plataforma remota Google Meet frente a los estudiantes participantes. Los momentos de seguimiento proporcionaron la creación de vínculos entre los monitores y los alumnos beneficiados por el programa de seguimiento, y esto facilitó el proceso de enseñanza-aprendizaje de la asignatura en cuestión. Durante el seguimiento fue posible a través de la revisión continua de los contenidos la creación de una base sólida de conocimientos, así como la búsqueda constante de la actualización de la información y el uso de las tecnologías, deduciéndose así la gran importancia del programa de seguimiento para los alumnos del colegio. La experiencia representó como una actividad relevante para los monitores, ya que permitió el crecimiento académico, profesional y personal, así como podemos observar varias ganancias al público participante.

Palabras clave: Estudiantes de enfermería; Ensenãnza; La educación en enfermería; Educación a distancia.

\section{Introdução}

A enfermagem abrange uma gama de conhecimentos técnico-científicos, elaborados através de práticas sociais, éticas e políticas que acontecem por meio do ensino, extensão e pesquisa, sendo realizada a partir da contribuição de serviços ofertados ao indivíduo, a família e a comunidade em combinação ao cenário em que estes encontram-se inseridos (Nunes, 2012).

Segundo o que rege a Lei das Diretrizes e Bases da Educação Nacional - LDB, a monitoria no quesito universitário 
possibilita que os discentes da educação de ensino superior possam executar atividades de ensino e pesquisa dentro das instituições praticando a função de monitor, desde que em consonância com o projeto político pedagógico da instituição (Brasil, 1996).

A Cristo Faculdade do Piauí (CHRISFAPI) possui o Programa de monitoria instituído pela resolução 005/08 de 12 de dezembro de 2008 a qual dar disponibilidade as normas para o processo de seleção e admissão de discentes monitores e de outras providências. $\mathrm{O}$ aluno diante da monitoria não substitui o professor, mas contribui diretamente com o processo ensino aprendizagem. A monitoria é uma atividade que aprecia a participação do aluno-monitor em momentos teóricos e práticos juntamente aos discentes com o intuito de aprofundar o conteúdo e em busca de um desenvolvimento associado à prática docente.

O curso bacharelado em enfermagem da Cristo Faculdade do Piauí, em seu Projeto Político Pedagógico conjectura para a disciplina de Semiologia e Semiotécnica uma carga horária de 60 horas de aula teórica e 40 horas de aula prática, na qual tem como disciplina de pré-requisito Fisiologia Humana, contudo, são nestes momentos teóricos e práticos que contemplam a colaboração do estudante monitor.

A disciplina de Semiologia e Semiotécnica, por sua parte, ampara os procedimentos técnicos executados no laboratório e no carregar de toda vida do profissional enfermeiro. Sendo assim, os momentos práticos desta disciplina é como um "divisor de águas" para o acadêmico do curso de enfermagem, na qual o graduando se depara com o seu primeiro contato diante das técnicas propedêuticas, com equipamentos e materiais, que até o presente momento certamente pode lhe causar estranheza, e que postulam habilidades e conhecimentos essenciais. Sobretudo, o que é perceptível diante do público cursante da disciplina é uma preocupação em ter o entendimento diante das práticas e poder executá-las de modo correto, assim tornando por consequência a prática da enfermagem de modo seguro e preciso (Korb et al., 2015; Steindorff et al., 2017).

O monitor condiz com o estudante de graduação do curso de enfermagem selecionado para executar atividades técnico-didáticas junto à determinada disciplina. Para a realização das atividades, o monitor deve fazer um planejamento em parceria com o professor orientador do conteúdo programático, e este deve ser aprovado mediante à coordenação pedagógica, entretanto, é providenciado a divulgação diante dos alunos e organizado a estrutura correspondente ao funcionamento do momento de monitoria. Essa atividade que concerne com o planejamento inicial de cada momento da monitoria é de extrema relevância, pois é neste ato que o monitor inicia o processo de autonomia e confiança em função aos conhecimentos obtidos (Andrade et al., 2018; Viana \& Da Silva, 2018).

Nessas circunstâncias, o aluno-monitor torna-se um facilitador e mediador da aquisição de conhecimento do outro acadêmico. É um protagonista que interage e forma vínculo com colegas de curso e professores. Assim, a relação de ensino entre o monitor e o acadêmico se estipula de forma mútua, onde os dois lados são contribuintes no processo de aprendizagem, e o aluno-monitor soma no sentido de instigar o crescimento crítico do aluno receptor da monitoria, bem como, relata ainda que a atividade de monitoria contribui como um auxílio pedagógico. A literatura também enfatiza que o projeto de monitoria favorece uma maior confiança na execução de procedimentos pelos discentes (Oliveira, Rocha \& Pereira, 2014; Nunes, 2012; Diodato et al., 2017).

Diante do exposto acima, no presente estudo tem como objetivo relatar a experiência vivenciada por graduandos do curso de enfermagem da Cristo Faculdade do Piauí (CHRISFAPI), no decorrer das atividades promovidas nas ações de monitoria da disciplina de Semiologia e Semiotécnica, ponderando em relação a notoriedade deste instrumento metodológico que impulsiona a prática acadêmica e proporciona subsídios para o profissional de enfermagem.

\section{Metodologia}

O presente trabalho trata-se de um estudo descritivo, de natureza qualitativa, do tipo relato de experiência, 
desenvolvido com base na vivência acadêmica na monitoria da disciplina de Semiologia e Semiotécnica, que no curso de bacharelado em Enfermagem da Cristo Faculdade do Piauí (CHRISFAPI) é oferecida a discentes do IV semestre. As atividades aconteceram no município de Piripiri-PI, no período de agosto a dezembro de 2020, correspondendo ao semestre 2020/2. O relato de experiência tem como finalidade a descrição de experiências vivenciadas de determinada população ou fenômeno (Gil, 2007; Silva \& Menezes, 2001).

Para de Moura Evêncio et al., (2019), a pesquisa é definida como o processo sistemático e formal do método científico, que a partir de um problema real, é possível descobrir caminhos para resolução deste problema. Já a pesquisa com abordagem qualitativa visa o direcionamento para o desenvolvimento de estudos buscando respostas que permitam descrever, entender e interpretar fatos sobre lugares, pessoas e processos interativos por meio da relação direta do pesquisador com o caso estudado (Proetti, 2018). Além disso, os métodos qualitativos são imprescindíveis para o pesquisador saber interpretar com suas opniões quanto ao fenômeno estudado (Pereira et al., 2018).

Diante disso, foi feito um levantamento bibliográfico com seleção dos artigos na Biblioteca Virtual em Saúde (BVS), por intermédio dos bancos de dados: Literatura Latino-Americana e do Caribe em Ciências da Saúde (Lilacs) e Medical Literature Analysisand Retrieval System on-line (Medline), sendo utilizados os seguintes descritores: Estudantes de enfermagem; Ensino; Educação em enfermagem e Educação a distância, no qual foram selecionados por meio dos Descritores em Ciências da Saúde (DeCS).

Para os critérios de inclusão, foram utilizados artigos publicados nos idiomas português, inglês e espanhol, disponíveis na íntegra, completos e que abordassem a temática sobre monitoria no contexto acadêmico e monitoria em modalidade de ensino a distância. Excluiu-se artigos não condizentes com a temática e àqueles que estavam incompletos.

Em relação a disciplina Semiologia e Semiotécnica, esta possui carga horária de 60 horas e trabalha com conhecimentos acerca da avaliação do estado de saúde dos indivíduos em seu ciclo vital, além de exame físico com ênfase na integração enfermeiro/paciente, coleta de dados subjetivos e objetivos e análise de dados coletados, além do julgamento clínico e interpretação de testes e/ou exames, bem como estuda a Sistematização da Assistência de Enfermagem (SAE).

Sobre o processo de seleção, a instituição estabelece que somente alunos regularmente matriculados podem se inscrever, além disso precisam ter cursado na própria instituição a disciplina na qual pretende desenvolver a monitoria, tendo atingido, no mínimo, uma média sete e que não tenha quaisquer pendências diante da faculdade.

Salienta-se que devido a Pandemia COVID-19, a seleção da monitoria foi realizada através de uma entrevista online e da análise do coeficiente de rendimento na disciplina Semiologia e Semiotécnica, a entrevista consistia em perguntas a respeito da docência e disponibilidade a fim de evitar convergência de horários entre os alunos monitorados e os monitores.

Sobre o programa de monitoria da faculdade, ele tem como principal objetivo estimular a iniciação à docência, sendo necessário a disponibilidade de 2 horas semanais para o desenvolvimento das atividades. A turma da disciplina era composta por 35 alunos do $4^{\circ}$ período do curso de Enfermagem.

\section{Resultados e Discussão}

\subsection{Atividades realizadas durante a monitoria de semiologia e semiotécnica}

A monitoria é uma modalidade de ensino-aprendizagem que incentiva o aluno para as atividades de ensino, pesquisa e extensão durante a graduação. É vista como uma ferramenta para o aperfeiçoamento do ensino, por meio da implantação de novas práticas e experiências educativas que visam o fortalecimento do vínculo entre teoria e prática, assim como a inserção curricular em diferentes perspectivas e saberes (Cavalcanti \& Marinho, 2018; Matoso, 2014).

Diante disto, o monitor deve estar disposto e apto para contribuir no desenvolvimento de ensino-aprendizagem do aluno monitorado, sendo um elo entre o docente e esse aluno. Entretanto, esse processo é somente eficaz se o aluno que tem 
acesso as monitorias demonstre interesse e curiosidade pela construção de seu conhecimento, assim, desfrutando de oportunidades que surgem nas instituições de ensino. No entanto, algumas vezes, é possível observar que alguns alunos negligenciam esse reforço ofertado pelo monitor (de Sousa Cunha \& da Costa, 2019).

Em decorrência da Pandemia COVID-19, as aulas da Cristo Faculdade do Piauí estão sendo na modalidade remota desde o início de 26 de março de 2020, isto é, a instituição segue cumprindo a determinação dos órgãos governamentais. Dessa forma, as atividades da monitoria também tiveram que se adaptar as novas normas estabelecidas.

As principais atividades desenvolvidas durante a monitoria se pautaram nos plantões tira-dúvidas por meio da ferramenta de ensino Google Meet. Ressalta-se que a internet trouxe inúmeras vantagens, dentre elas, a de oferecer outros métodos de ensino-aprendizagem. Com isso, os acadêmicos puderam ter acesso aos conteúdos repassados na monitoria no momento em que estivessem mais livres, portanto, tendo maior aproveitamento e rendimento da disciplina.

Além disso, destaca-se que os plantões proporcionaram a criação de vínculo entre os monitores e alunos beneficiados pelo programa de monitoria, e isso facilitou o processo de ensino-aprendizagem da disciplina em questão. Sob esse viés, essa dinâmica e parceria entre monitor e aluno influenciou positivamente no desempenho da turma, tanto nas avaliações teóricas quanto nas práticas remotas.

Além dos plantões tira-dúvidas os monitores disponibilizaram material complementar e questionários com os assuntos discutidos durante as atividades. Ademais, também realizaram vídeos com práticas dos respectivos assuntos solicitados pela professora da disciplina, o que facilitou ainda mais a compreensão dos alunos em relação aos assuntos passados durantes as aulas.

Por fim, com o objetivo de auxiliar no repasse dos conteúdos, os monitores elaboraram roteiros para nortear os discentes nos estudos dos assuntos programados na disciplina de Semiologia e Semiotécnica, como Introdução a Semiologia; Técnicas propedêuticas para realização do exame físico; Exame físico geral; Teorias de enfermagem e Sistematização da Assistência de Enfermagem; Semiologia dos sistemas digestório, neurológico, cardiovascular, respiratório, tegumentar, geniturinário, músculo esquelético e semiologia da cabeça e pescoço, bem como avaliação antropométrica.

\subsection{Importância da monitoria para a formação acadêmica e atuação profissional}

As monitorias possibilitam o aperfeiçoamento diante da disciplina, assim como do potencial acadêmico, das habilidades técnicas de enfermagem, a segurança, a capacidade de observação, maior afinidade com os métodos semiológicos, conhecimento obtido durante o ensino da monitoria, memorização dos conteúdos estudados, correlacionando sempre, os momentos teóricos com a prática (da Silva \& de Belo, 2012; Schmitt et al., 2013).

Desse modo, os acadêmicos monitores e os estudantes da disciplina de Semiologia e Semiotécnica ganham maior confiança, assim como para quando ter o contato com o paciente e tiver que executar os aprendizados de Semiologia e Semiotécnica, dando maior facilidade para os momentos práticos dos estágios nas Unidades Básicas de Saúde - UBS, setores dos hospitais, dentre outras situações (Ziani, Zuge \& Harter, 2019).

Apesar das monitorias terem sido executadas de modo à distância em razão da atual situação pandêmica, os acadêmicos agregam fatores positivos em relação a sua atuação como estudante ou mesmo no futuro como profissional, isso no que concerne a redução da ansiedade, medo, e foram beneficiados no suprimento nas dúvidas no conteúdo revisado. Houve a aplicação assertiva das técnicas de enfermagem, e assim possibilitou ao acadêmico uma maior segurança em uma possível prestação de cuidados ao cliente.

Hagg et al., (2008) em sua pesquisa na Universidade do Vale do Rio dos Sinos (UNISINOS), todos os participantes do estudo inferiram que há uma influência positiva da monitoria, o estudo mostrou que 47\% relataram maior conhecimento, $40 \%$ melhor atuação nos estágios e 13\% enfatizaram a promoção de confiança. Diante dos dados, os autores ressaltaram que 
monitoria contribui também nos quesitos psicológicos, pois as práticas possibilitadas dirimem a ansiedade e o universitário consegue executar o que é proposto com segurança.

Com a finalidade de autenticação do conhecimento aos acadêmicos, as atividades da monitoria seguiam-se um roteiro base para os slides a fim de direcionar o estudo da fundamentação teórica das técnicas estudadas. Segundo Serafim et al., (2007), os materiais didáticos planejados para as monitorias são de extrema relevância, pois proporcionam um atendimento de qualidade, e a curto prazo possibilita a visualização da técnica e fazendo uma "ponte" para a execução da mesma.

Nas monitorias foi possível ter uma otimização das aulas de Semiologia e Semiotécnica, dando um auxílio para o docente nas diversas atividades da disciplina. Foi notável uma procura considerável da monitoria, em virtude de mesmo havendo a conclusão da disciplina, as técnicas de enfermagem adquiridas, serão utilizadas em toda a vida de estudante e se propagam pela vida profissional.

O aluno-monitor desenvolve uma análise crítica diante do processo de ensino e aprendizagem ao ter contato com as mais diversas metodologias desenvolvidas pelos docentes das disciplinas. Isso proporciona a criação do seu próprio método de ensino, no fato do monitor futuramente seguir a vida docente, esse contato breve somará de forma significativa no perfil do profissional que o acadêmico irá ter (Nunes, 2012; Ziani, Zuge \& Harter, 2019).

$\mathrm{Na}$ execução da monitoria foi possível por intermédio da revisão contínua dos conteúdos a criação de uma base sólida de conhecimento, bem como, pela busca constante de atualização de informação e o uso de tecnologias, assim sendo inferido a tamanha importância do programa de monitoria para os discentes da faculdade. Serafim et al., (2007), ressalta que o processo de edificação do conhecimento entre docentes e monitores, surgem novas ideias, com o intuito de melhorias, ademais, esta atividade suplementar, tem a finalidade de auxiliar todos os participantes.

Para os discentes monitores, houve um grande crescimento pessoal, sendo que estes tiveram a oportunidade do desenvolvimento de habilidades de liderança e de entrar em contato direto com as atividades de ensino, proporcionando ainda uma maior interação com os estudantes e professor. A monitoria impactou diretamente no quesito de desenvolvimento pessoal e profissional dos monitores, aprimorando os conhecimentos da graduação, mais especificamente na área de Semiologia e Semiotécnica.

\section{Conclusão}

A monitoria representou-se como uma atividade relevante para os monitores, uma vez que possibilitou o crescimento acadêmico, profissional e pessoal. Com isso, por meio dessa experiência desenvolvida na monitoria de Semiologia e Semiotécnica, pode-se perceber que os monitores puderam aprimorar, elaborar e implementar atividades de ensino e pesquisa.

Além disso, observa-se que essas atividades se configuram como uma experiência importante para o aprendizado dos monitores, visto que é uma oportunidade para conhecer e vivenciar, de maneira mais próxima, a realidade do processo de ensino e aprendizagem sob a luz da docência, além de proporcionar uma troca de saberes entre aluno e monitor.

Ressalta-se ainda que os desafios foram superados ao longo da monitoria, como por exemplo, as atividades realizadas de maneira remota devido a Pandemia Covid-19. Enfatiza-se, portanto, que a experiência como monitor de tal disciplina exigiu organização, compromisso e responsabilidade, além disso, promoveu troca de conhecimentos significativos no processo de formação acadêmica entre monitores e alunos monitorados.

Portanto, sugere-se que novos relatos de experiência sejam realizados, especialmente nesse momento (ensino remoto) que as instituições de ensino e os próprios acadêmicos estão vivenciando, uma vez que é possível utilizar as estratégias de ensino que são desenvolvidas e implementadas nesse tipo de estudo. Além do mais, cabe ao monitor se comprometer diante de várias adversidades, visto que esses atributos the confere competência na formação acadêmica e, consequentemente, seja capaz de sugerir soluções para as diversas situações de trabalho enquanto futuro profissional. 


\section{Referências}

Andrade, E. G. R. D., Rodrigues, I. L. A., Nogueira, L. M. V., \& Souza, D. F. D. (2018). Contribuição da monitoria acadêmica para o processo ensinoaprendizagem na graduação em enfermagem. Revista Brasileira de Enfermagem, 71, 1596-1603. http://dx.doi.org/10.1590/0034-7167-2017-0736

Brasil, Constituição. (1996). Lei $n^{\circ}$ 9.394, de 20 de dezembro de 1996. Estabelece as diretrizes e bases da educação nacional. Diário Oficial da União, 134, 248 .

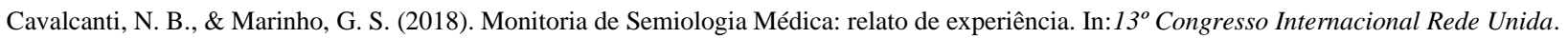

Diodato, I. D. T., Primão, J. C. M., Vieira, S. A. M., Venazzi, C. B., \& de Paula Alves, M. A. (2017). A ação da monioria de semiologia na vida acadêmica dos discentes do curso de enfermagem. In: IV Semana Acadêmica de Sinop.

Gil, A.C. (2007). Como elaborar projetos de pesquisa. Atlas.

Haag, G. S., Kolling, V., Silva, E., Melo, S. C. B., \& Pinheiro, M. (2008). Contribuições da monitoria no processo ensino-aprendizagem em enfermagem. Revista Brasileira de Enfermagem, 61(2), 215-220. http://dx.doi.org/10.1590/S0034-71672008000200011.

Korb, A., Nothaft, S. C. D. S., Silveira, A. D. M., \& Brum, M. L. B. (2015). Atividade integrativa das disciplinas de Microbiologia com Semiologia e Semiotécnica: higienização das mãos. Rev. Saúde Pública St. Catarina, 80-97.

Matoso, L. M. L. (2014). A importância da monitoria na formação acadêmica do monitor: um relato de experiência. CATUSSABA-ISSN 2237-3608, 3(2), 7783.

Moura Evêncio, K. M., Teixeira, S. L., Rodrigues, K. G. F. C., Feitosa, F. A., \& da Silva Fontes, W. J. (2019). Dos Tipos de Conhecimento às Pesquisas Qualitativas em Educação/From Knowledge Types to Qualitative Education Research. ID on Line Revista de Psicologia, 13(47), 440-452.

Nunes, V. M. A. (2012). Monitoria em semiologia e semiotécnica para a enfermagem: um relato de experiência. Revista de Enfermagem da UFSM, 2(2), 464471. https://doi.org/10.5902/217976923212

Oliveira, L. A., Rocha, J. E., \& Pereira, V. S. (2014). Fatores que levam o aluno a engajar-se em programas de monitoria acadêmica de uma instituição de ensino superior. Revista Interfaces: Saúde, Humanas e Tecnologia, 2(4).

Pereira, A. S., Shitsuka, D. M., Parreira, F. J., \& Shitsuka, R. (2018). Metodologia da pesquisa científica. UFSM

Proetti, S. (2018). As pesquisas qualitativa e quantitativa como métodos de investigação científica: Um estudo comparativo e objetivo. Revista Lumen-ISSN: 2447-8717, 2(4).

Schmitt, M. D., Ribeiro, M. C., Adamy, E. K., Brum, M. L. B., \& dos Santos Zanotelli, S. (2013). Contribuições da monitoria em semiologia e semiotécnica para a formação do enfermeiro: relato de experiência. Cidadania em Ação: Revista de Extensão e Cultura, 7(1).

Serafim, D., Ichisato, S. M. T., Correa, D. A. M., Marino, M. M., Ciaciare, B. C., \& Corrêa, J. L. (2007). Estratégias de ensino na monitoria de saúde da mulher e da criança do curso de enfermagem da Universidade Estadual de Maringá. Ciência, Cuidado e Saúde, 6, 474-480. https://doi.org/10.4025/cienccuidsaude.v6i0.5360.

Silva E.L., \& Menezes E.M. (2001). Metodologia da pesquisa e elaboração de dissertação. Florianópolis: Laboratório de Ensino a Distância da UFSC.

Silva, R. N., \& de Belo, M. L. M. (2012). Experiências e reflexões de monitoria: contribuição ao ensino-aprendizagem. Scientia Plena, 8(7).

Silva, V. B., \& Harter, J. (2017). A importância da monitoria de semiologia em enfermagem para o discente-monitor: relato de experiência. Anais do Salão Internacional de Ensino, Pesquisa e Extensão, 8(1).

Sousa Cunha, L., \& da Costa, F. N. (2019). A importância da monitoria na formação acadêmica do monitor: um relato de experiência. Encontro de Extensão, Docência e Iniciação Científica (EEDIC), 4(1).

Steindorff, G., Simon, B. S., de Oliveira Junior, S. B., Garcia, R. P., Jaques, J. A., \& Lima, B. G. (2017). Monitoria acadêmica no componente curricular de Semiotécnica em Enfermagem: Relato de experiência. Anais do Salão Internacional de Ensino, Pesquisa e Extensão, 8(1).

Viana, F. A. M., \& Da Silva, J. F. (2018). Metodologia ativa na monitoria de semiologia e semiotécnica: uma vivência acadêmica. Conexão Fametro 2018: Inovação e Criatividade, 1-6.

Ziani, J., Zuge, B. L., \& Harter, J. (2019). Análise de pré e pós teste em monitoria de semiologia em enfermagem. Anais do Salão Internacional de Ensino, Pesquisa e Extensão, 11(1). 